\title{
NIVEL DE CONOCIMIENTO QUE TIENEN LOS ALUMNOS DE NIVEL MEDIO SUPERIOR EN TÓPICOS FINANCIEROS SOBRE GASTO Y CRÉDITO, AHORRO E INVERSIÓN Y LA ADMINISTRACIÓN DEL DINERO
}

\author{
Belém Alejandra Contreras Rodríguez \\ UCC Business School at Universidad Cristóbal Colón, Veracruz México \\ E-mail: alejandrac46@hotmail.com \\ Arturo García-Santillán \\ UCC Business School at Universidad Cristóbal Colón, Veracruz México \\ E-mails: arturogarciasantillan@yahoo.com.mx \\ Elena Moreno-García \\ UCC Business School at Universidad Cristóbal Colón, Veracruz México \\ E-mails: elenam@ucc.mx \\ https://doi.org/10.17060/ijodaep.2017.n1.v2.962
}

Fecha de Recepción: 14 Marzo 2017

Fecha de Admisión: 1 Abril 2017

\section{RESUMEN}

El propósito de este trabajo se centra en determinar el nivel de conocimiento que presentan los estudiantes de educación media superior en tópicos financieros como gasto y crédito, ahorro e inversión y la administración de dinero. Los participantes fueron 368 alumnos de diferentes bachiIleratos en tres municipios del Estado de Veracruz, mismos que fueron seleccionados a partir del cálculo de la muestra estratificada llevada a cabo. El instrumento es una escala de 31 ítems de opción múltiple en forma de casos en donde solo hay una respuesta correcta del total de las 4 opciones. El instrumento mostró una fiabilidad y consistencia interna alfa de Chronbach aceptable (>0.7). Los resultados nos llevan a pensar que hay un desconocimiento en el alumnado sobre la utilidad de las pensiones, los temas relacionados a los seguros, el impacto que la inflación genera en los sueldos y salarios de las personas, incluso un dato interesante es que, aunque se conoció que hay un conocimiento sobre los canales básicos de ahorro, denotan ausencia de conocimiento hacia los instrumentos de inversión, especialmente a los que por su naturaleza se asocian a los mercados de capitales

Palabras clave: Inclusión financiera, educación financiera, conocimiento financero.

\section{ABSTRACT}

The aim of this paper is focused in measure the level of knowledge they have high school stu- 
dents about financial topics such as: spending and credit, savings and investment and money management. The participants were 368 students of different high schools in three municipalities of the State of Veracruz, México who were selected from of the stratified sample. The questionnaire is a scale that includes 31 items of multiple choices in the form of cases where there is only one correct answer of the total four options. The instrument showed acceptable Chronbach alpha reliability and internal consistency (>0.7). The results lead us to think that there is a lack of knowledge about the usefulness of pensions, the issues related to insurance, the impact of inflation on salaries of people workers, even an interesting fact is that although it was known that there is a knowledge about the basic channels of saving, they denote lack of knowledge towards the investment instruments, especially those that by their nature are associated with the capital markets.

Keywords: Financial inclusion, financial literacy, financial knowledge

\section{PLANTEAMIENTO DEL PROBLEMA}

Hoy en día, el tema de Inclusión financiera (IF), ha acaparado la atención de los países en todo el orbe, es decir, se ha llevado a tema de agenda mundial, ejemplo palpable de ello es el Grupo de los 20 países lideres (G-20), que desde el 2009 ha realizado intercambio de compromisos para incrementar el acceso a la población de nivel bajo a los servicios y productos financieros, creando un subgrupo especial para este tema, como línea de tiempo se puede mencionar que en 2010 se concretaron esos compromisos en un intercambio de experiencias vividas y modelos planeados, en donde señala textualmente en su reporte Principles and Report on Innovative Financial Inclusion from the Access through Innovations (G-20, 2010) que:

"la inclusión financiera es una condición necesaria para la reducción de la pobreza. No solo otorga a los segmentos más pobres de la población la posibilidad de incrementar o estabilizar sus ingresos, sino que también los provee de una mayor resistencia a los choques económicos y de la oportunidad de poseer activos. Contribuye al desarrollo del sector financiero, lo que incentiva el crecimiento económico mediante la movilización de ahorro y la inversión en el crecimiento del sector productivo. El crecimiento económico también favorece indirectamente la disminución de la pobreza"(G-20,2010).

Lo anterior, pone sobre la mesa de trabajo del subgrupo de expertos en inclusión financiera del propio G-20 las preocupaciones derivadas como consecuencias que han dejado las crisis financieras en los paises desarrollados y en desarrollo, pero mas que las consecuencias, se ha discutido acerca de, cuáles han sido las causas que llevaron a los paises a vivir esos problemas economicos y cual es el camino al que deben orientar sus políticas públicas para incrementar la inclusión financiera en su población (G-20, 2012).

Para promover el intercambio global de modelos implementados en distintos países, el G-20 adopta en 2010 a la Alianza de Inclusión financiera (AFI), quien había iniciado en 2008 como una fundación particular con 6 países miembros, entre ellos México, se integran a la misma para conformarla como una red desde la que se ejecutan los planes de acción para elevar la IF (AFI, 2013).

Mediante los trabajos realizados con los expertos en finanzas de los 90 países miembros del G20, la AFI ha logrado identificar que alrededor de 2,500 millones de personas de la población mundial no está "bancarizada".

La AFI considera tres niveles de acción para mejorar la inclusión financiera, a través de alianzas público-privadas. El nivel macro, en el que consideran los entorno y regulación para promover el acceso a servicios financieros; el nivel meso, donde se integra el soporte a la oferta y demanda de 
Ios servicios financieros tales como sistemas de pagos y protección al consumidor; y el nivel micro en el que se realizan acciones para apoyar la red de participantes del sector financiero incluyendo la cobertura geográfica, y para mejorar el acceso a hogares de bajo nivel económico mediante cofinanciación y asistencia técnica a servicios financieros.

Por su parte el Banco Mundial (BM) en un reporte denominado Global Financial Development Report, (BM, 2014) ha centrado su discusión en la relación que existe entre la IF y el desarrollo económico, reuniendo evidencia empírica sobre el impacto de la IF en el desarrollo económico y la pobreza, considerando que, dependiendo de la etapa de desarrollo, el grado de inclusión financiera difiere de un país a otro.

Para mostrar dicha relación, el BM presenta los resultados de un sondeo realizado a los participantes del sector financiero, como representantes de bancos centrales, ministros de finanzas, representantes de ONG's, así como académicos involucrados de 54 países clientes del BM, en donde se observó que a nivel mundial 2.5 millones de adultos hace uso de algún servicio financiero. La IF reduce considerablemente la pobreza trayendo beneficios cuantificables que van desde pagos básicos, ahorros y servicios de seguros al sector pobre, y para las empresas el acceso a los servicios financieros se asocia con la innovación, creación de empleo y sobretodo crecimiento. 1

En su momento Lusardi $(2006,2008,2010)$ ha referido en múltiples estudios, que los individuos tienen un conocimiento escaso sobre términos financieros, de ahí que, desde las acciones que la Organización de las Naciones Unidas (ONU) se ha creado en 2012 el movimiento Child and Youth Finance International, respaldado por el Fondo de las Naciones Unidas para la Infancia (UNICEF), el cual tiene como premisa que los jóvenes de hoy, son futuros actores económicos, quienes tomarán decisiones dentro de las finanzas, dirigiendo el sentido de las economías mundiales, de ahí la importancia de brindar a los jóvenes y niños ambientes, económica y socialmente adecuados para que se generen las condiciones de un crecimiento prospero para la economía, así como las competencias para que esas decisiones tengan un impacto positivo en la vida de los ciudadanos y de su nación (Child and Youth Finance Internacional, 2012).

Desde el ámbito global es grande el esfuerzo y compromiso de los países, a través de sus Bancos Centrales y aquellos expertos encargados del diseño de las políticas de estado, por aumentar el acceso a los servicios financieros de la población, de ahí que, se han diseñado estrategias que favorezcan este fin, ejemplo de ello, las relativas al plano económico y financiero de dichos países. Es por ello que en el 2013 se publica el documento titulado: "Ubicando la inclusión financiera en el mapa mundial. Informe sobre avances de la Declaración Maya 2013" el cual recoge información relevante sobre los acuerdos y compromisos de los miembros de la Alianza para la Inclusión Financiera cuyo propósito es el de favorecer la IF. 2

Pero en escenarios nacionales, recordemos que en el 2011 fue adoptada en México la Declaración Maya (única en su tipo) y se ha venido convirtiendo en un gran esfuerzo de colaboración entre sus miembros, con un rápido crecimiento e impacto mundial.

\subsection{Caso Mexico}

Para el caso de México, una de las preocupaciones plasmadas en el Plan Nacional de Desarrollo 2007-2013, es lograr la democratización del sistema financiero mexicano sin arriesgar su solvencia, fortaleciéndolo como detonador de crecimiento, equidad y desarrollo económico del país, lo cual ha sido una preocupación transexenal, que se ha continuado en el Plan 2013-2018. El objetivo primordial, es aumentar la inclusión financiera a la mayor población posible, armonizando los trabajos de las distintas dependencias para contribuir desde cada sector, al logro del mismo objetivo (PND, 2007 y 2013). 
Por su parte la Secretaria de Hacienda y Crédito Público (SHCP) quien tomando esto como premisa, ha dirigido sus esfuerzos para generar estrategias con el objetivo de incrementar la inclusión financiera, estrategias que incluyen cinco objetivos: favorecer la sustitución del dinero en efectivo por dinero electrónico; impulsar el uso de medios de pago electrónicos; utilizar más y de mejor manera nuevas tecnologías que permitan el acceso a servicios financieros a la población; lograr la bancarización total de programas sociales y nóminas gubernamentales, y apoyar el acceso al financiamiento para personas que usualmente no han tenido acceso al crédito, para que puedan generar historiales crediticios. Mediante estas estrategias, la política de la secretaria es incrementar el acceso a los servicios financieros básicos, lo cual permite a las familias nivelar su consumo e incrementar la inversión en educación y salud, así como asegurarse ante posibles eventos que ponen en riesgo su patrimonio.

Como seguimiento a la propuesta de la AFI de fomentar la alianza de gobierno-sector privado y actores económicos, y con la finalidad de lograr una mayor especialización en temas de inclusión financiera, así como generar y proveer de información útil para los organismos involucrados en el sector financiero, es que en el 2011 se crea el Consejo Nacional de Inclusión Financiera (CONAIF), como un organismo de consulta, asesoría y coordinación para proponer medidas de planeación, formulación, instrumentación, ejecución y seguimiento de una política nacional para la IF.

EI CONAIF ha llevado a cabo la encuesta nacional de inclusión financiera en 2012, donde encontró que 25 millones de adultos tienen al menos un producto de ahorro formal; de éstos, 24 millones poseen alguna tarjeta de débito; aproximadamente el $20 \%$ de los ciudadanos en el territorio nacional no emplea servicios financieros formales; $60 \%$ usa tandas o préstamos de fuentes no formales y cerca de la mitad de los municipios rurales no cuentan con una sucursal bancaria (CONAIF, 2013).

El interés de México por incrementar la inclusión financiera en cada región del país, como cimientos para un mejor desarrollo económico, ha sido palpable en diferentes participaciones a través de escenarios mundiales, como el seminario internacional de "Inclusión Financiera responsable y exitosa: modelos internacionales" que en 2013 fue presidido por el Banco de México, quien al referirse a temas de IF, propone que se debe reconocer obligatoriamente las experiencias vividas en crisis financieras recientes y cada vez más se pronuncia por asociar los índices de pobreza con una baja inclusión financiera, siendo esta, un componente central del desarrollo financiero ya que permite que exista una mayor cantidad de recursos para la inversión y que se haga una asignación más eficiente de los recursos ${ }^{3}$.

Si bien el Banco de México propone marcos regulatorios para atraer la confianza de los consumidores hacia el uso de servicios y productos financieros y apoya programas que permitan acercar estos servicios a las poblaciones de difícil acceso, así como también el apoyo que brinda a la banca privada para la generación de nuevos productos asequibles a niveles más bajos, los esfuerzos para aumentar la IF se ven concretados con la creación del Museo Interactivo de Economía (Mide) para difundir conocimientos básicos de economía, con el fin de promover a través de este, la educación financiera a la población en general, resaltando sus acciones hacia la población joven. Sin embargo el rol principal que desempeña el Banco de México para la IF continua siendo como el motor que permite el mantenimiento de la estabilidad de precios.

Como banca privada, sumándose a estos esfuerzos, se puede hacer mención del grupo BBVA Bancomer, quien al analizar la encuesta que elaboran la CNBV a través del CONAIF, concluye los factores que desde el lado de la demanda determinan los obstáculos de acceso a los servicios financieros, los cuales son: ingresos insuficientes, autoexclusión, razones personales y barreras de acceso.

El grupo BBVA ha contribuido con el Banco de México a fomentar el uso de telefonía móvil en los servicios financieros, focalizando especial interés en la educación financiera, desarrollando el 
programa "adelante con tu futuro", programa que le ayuda a las personas a usar adecuadamente Ios servicios financieros, para recorrer todo el proceso educativo completo: con la esencia de pasar de la "conciencia" al "saber", pero sobre todo del "saber" al "saber hacer"; en otras palabras, ayudan a la gente no solo a reconocer por qué es importante que incremente su cultura financiera, sino compartirle el conocimiento y que desarrollen las habilidades, para usar el ahorro y el crédito a su favor.

Es claro, que si bien la educación es necesariamente deseable, no debe ser el único medio para alcanzar mejoras en las prácticas financieras, pero si es un fragmento que influye en la Inclusión Financiera, ya que, si bien, la IF es el acceso universal de la población a los servicios y productos financieros que ofrecen los sistemas financieros, entonces debemos cuestionarnos sobre:

¿Cuál es el nivel de conocimiento que tienen los estudiantes de educación media superior, en tópicos de administración de dinero, ahorro e inversión y finalmente en gasto y crédito?

Para responder a esta interrogante, es necesario fijar como objetivo: Determinar el nivel de conocimiento que tienen los estudiantes de educación media superior, en tópicos de administración de dinero, ahorro e inversión y finalmente en gasto y crédito. Con esta consideración es factible que puedan estar incluidos en el fenómeno de Inclusión Financiera.

Por lo antes expuesto, ahora se analiza y discute en el siguiente apartado el estado del arte que define la situación actual de la inclusión financiera desde su realidad teórica y la evidencia empírica que se ha generado en torno a este tema que se investiga.

\section{li. Fundamentación}

Los estudios que se han generado en relación al tema de Inclusión Financiera (G-20, OCDE, Banco Mundial, Unicef, CNBV, CONAIF,), son en esencia holísticos, sin embargo en la medida en que se profundiza en este tema, se va tratando de explicar de manera holística en relación a los factores que contribuyen a su incremento, como lo han referido Atkinson, McKay y Kempson (2006), Lusardi (2008), Hastings y Tejeda (2008), Lusardi y Michell (2011).

En este sentido, son los jóvenes quienes finalmente tomarán las futuras decisiones financieras para su vida, relacionadas éstas a aspectos tales como: ahorro, incremento de patrimonio, créditos y en el mejor de los casos inversiones de negocios. Al respecto refiere Lusardi (2008) que menos de un tercio de los jóvenes poseen conocimientos básicos de los tipos de interés, la inflación y la diversificación del riesgo y además, si tomamos como referente sólido lo expuesto por el movimiento Child and Youth Finance de la UNESCO, se empieza a justificar la importancia por analizar el fenómeno de la IF en estas poblaciones jóvenes, como la que se ha propuesto investigar en este estudio.

Continuando con la revisión a la literatura especializada en el tema de IF, se han logrado identificar numerosos estudios en donde se analiza y discue este tema, es por ello que tomamos como referente inicial uno de los primeros trabajos seminales de Goldsmith (1959) y King y Levine (1993), quienes en sus investigaciones, lograron establecer a partir de sus resultados, que hay una correlacion positiva entre el crecimiento financiero y el desarrollo del sistema financiero. Ademas se robustece este dato, con los trabajos de otros autores como Demirguc-Kunt, Maksimovic (1998), quienes reportaron que cuando existe un avance en los índices económicos, entonces aumenta el uso de los productos y servicios financieros, especialmente en paises en desarrollo. Tambien refieren que el mercado bursátil debería apoyarse de las insituciones financieras, como los Bancos, a efecto de favorecer el incremento o mejora del desarrollo económico.

Diversos estudios han establecido la importancia que tiene para el desarrollo de los paises el mayor acceso a servicios financieros, demostrando que existe una alta correlación entre el creci- 
miento económico y la profundización del acceso a servicios financieros (Levine, Loayza y Beck, 2000). En la misma idea, Allen, Bartilro y Kowalewski (2006) refieren que el desarrollo economico de cada pais, crea una necesidad de productos y servicios financieros, señalan que el sistema financiero es el ente destinado a cubrir esas necesidades.

Partiendo de estos antecedentes se encuentran los trabajos de Caskey, Durán y Solo (2006), quienes definieron que el acceso a los servicios financieros, tales como: ahorro, pagos y crédito, puede hacer una diferencia positiva sustancial en la mejora de vida de la gente de bajos recursos económicos.

\section{li.1 Inclusión Financiera}

Uno de los primeros intentos por definir la inclusión financiera es el estudio presentado por Leyshon y Thrift (1995) quienes refieren que la IF incluye aquellos procesos que sirven para facilitar que diversos grupos sociales obtengan el acceso al sistema financiero formal. Según Sinclair (2001), la IF significa la posibilidad de acceder a los servicios y productos financieros de manera formal.

Por su parte el Banco Mundial la define como el acceso y utilizacion de los servicios financieros formales por parte de la poblacion excluida, lo cual se traduce en mayor crecimiento económico gracias a la expansion de la capacidad de consumo e incremento de la inversion. De ahí que La Alianza de Inclusión Financiera defiende la idea de que la IF se debe considerar como un rompecabezas conformado por herramientas que incrementan el ahorro interno, estimulan las PYMES y promueven el consumo privado. Dichas herramientas son: Entorno regulatorio propicio, Cobertura del sector (canales), Oferta de productos adecuados, Protección al consumidor y transparencia, Conocimientos financieros básicos para los consumidores.

Aun cuando la IF es el acceso y disponibilidad de una serie de productos y servicios financieros, resulta interesante lo que han manifestado Mehrota, Puhazhendhi, Nair y Sahoo (2009) en un estudio realizado en la India. En su reporte concluyen que el acceso a una cuenta de ahorro simple, fue la entrada a los servicios bancarios de la población que estudiaron, ya que señalan que la cuenta bancaria perse es un producto que incorpora valores como: la seguridad, la comodidad, la liquidez y la confianza que el producto representa para cubrir sus necesidades, de ahí que las personas de recursos bajos pueden acceder a una cuenta de ahorros de manera segura.

Un sistema financiero que facilita el acceso a diversas clases sociales, es deseable para facilitar la asignacion de los recursos de manera eficiente, asi como el acceso a los servicios financieros adecuados, contribuyen a un sistema financiero inclusivo el cual puede ayudar a reducir el crecimiento de las fuentes informales de crédito como lo menciona Sarma (2010). De ahí que Sarma define la inclusión financiera como un proceso que brinda el acceso y uso del sistema financiero formal para todos los miembros de una economía, esta definición hace hincapié en varias dimensiones de la inclusión financiera tales como: la accesibilidad, la disponibilidad y el uso del sistema financiero, estas dimensiones en su conjunto contribuyen a construir un sistema financiero incluyente.

Otras definiciones de la variable IF coinciden en el acceso a servicios financieros básicos como de ahorro, pagos y crédito, lo cual puede hacer una diferencia positiva sustancial en la mejora de vida de la gente pobre (Caskey et al., 2006; Dupas and Robinson 2009).

Como se ha referido anteriormente, para este estudio se retoma el concepto del G-20 (2010) en relación a la variable implicada, IF es una condición necesaria para la reducción de la pobreza. No solo otorga a los segmentos más pobres de la población la posibilidad de incrementar 0 estabilizar sus ingresos, sino que también los provee de una mayor resistencia a los choques económicos y de la oportunidad de poseer activos. Contribuye al desarrollo del sector financiero, lo que incentiva el 
crecimiento económico mediante la movilización de ahorro y la inversión en el crecimiento del sector productivo.

Finalmente retomamos lo que El Movimiento Child and Youth Finance (2012) ha señalado en torno a la IF, mismo que la definido el término de "la inclusión financiera" como la posibilidad de acceder a productos y servicios financieros asequibles, que se puedan emplear, que sean seguros y de absoluta confianza.

\section{II.2 EDUCACIÓN FINANCIERA}

De acuerdo con Ruiz (2011) La educación financiera es la fuerza motriz del desarrollo social, contribuyendo a procrear capital humano financieramente competente entre otras facultades; lo que lleva a las mejores decisiones para el beneficio de su economía. Es importante tener en cuenta que la educación financiera contribuye a tomar decisiones sabias, pero más allá de eso, Gnan, Silgoner y Weber (2007) y Mandell (2009) sugieren que contribuye al bienestar general de la economía. Según Gnan et al. (2007), la educación financiera suaviza el funcionamiento de los mercados financieros ya que se tienen mejores decisiones financieras de la población en su conjunto, dando pie a que esto contribuya a la reducción de la crisis económica y promuevan la estabilidad en el sistema financiero.

Después de que el Banco Mundial manifestará su preocupación en relación al tema de inclusión financiera, se han realizado diversos estudios, ejemplo de ello los trabajos de Lusardi y Michell (2006), quienes desarrollaron un estudio empírico para identificar el nivel de educación financiera en una población, de donde obtuvieron resultados que demuestran que los individuos tienen un conocimiento escaso sobre términos financieros, por su lado, Atkinson, McKay, Kempson y Collard, (2006) encontraron que la carencia de conocimiento de la población en materia financiera, repercute en las decisiones en temas financieros. Para su estudio utilizaron factores como la administración del dinero, planificación y la manera de seleccionar los productos financieros.

Lusardi en 2008 toma la encuesta de educación financiera del Health and Retirement Study (HRS) implementada en estados Unidos en 2004 y analiza los datos que le permiten concluir que bajos niveles de educación financiera disminuyen la capacidad de ahorrar, planificar y además a una modesta acumulación de riqueza. Otro estudio de Hastings y Tejeda (2008), refiere que las personas con menor conocimiento financiero tienen una menor probabilidad de planificar su jubilacion y de elegir fondos de pension con comisiones mas bajas.

Mientras tanto, Mandell (2009) refiere que las decisiones financieras de los consumidores tienen un efecto negativo en la economía, tales como una baja tasa de ahorro y la formación de capital, un bajo nivel de ahorro para el retiro, y una tasa más alta de la distribución desigual del ingreso y la riqueza.

En el plano de la discusión cientifica se encuentran estudios que inidcan que la educación financiera y económica sólo es eficaz en el corto plazo (un año) para los estudiantes, de acuerdo con Mandell (2008), quien realiza un estudio para la Jumpstart Coalition for personal literacy en donde encuesta a estudiantes de último grado de preparatoria, utilizando variables como: ingreso, administración del dinero, ahorro e inversión y gasto y crédito, en el cual comenta que los comportamientos financieros de los jóvenes no van a cambiar con el tiempo.

En una postura contraria se encuentra a Bernheim et al. (2001) y Tzu-Chin et al. (2007) quienes demostraron que un programa de educación financiera implementada en los años setenta y ochenta a estudiantes de nivel intermedio en los Estados Unidos, dio lugar a una mayor acumulación de la riqueza en el largo plazo (20 años), en comparación con los estudiantes que no asistieron a este programa. 
Pero en 2011 Lusardi y Mitchell encuentran efectos positivos entre la educación financiera y la capacidad para planificar, esto empata con lo que estableció Pinar, Heimann y Mylenko (2011) refiriendo a Dupas y Robinson quienes en el 2009 señalaron que el acceso a servicios financieros básicos como de ahorro, pagos y crédito puede hacer una diferencia positiva sustancial en la mejora de vida de la gente pobre.

Para el movimiento de la UNESCO denominado Child and Youth Finance International (2012), la alfabetización financiera es una destreza esencial para el bienestar social y económico, quienes, con el diseño de programas sobre educación financiera acordes a los niños y jóvenes constituyen un factor vital en el paso hacia esa ciudadania financiera que se adquiere con la edad adulta, lo cual favorece el desarrollo de las generaciones de ciudadanos económicos responsables hacia las economias de los países en donde se desarrollan y son parte de la población económicamente activa. De esta forma, las variables que retoma el movimiento Child and Youth Finance para medir el conocimiento en materia financiera son: ahorro, inversión, pensiones, créditos, deudas, gastos y presupuestos.

Derivado de la revisión a los fundamentos teoricos y empiricos, nos ha permitido identificar las variables que podrían estar implicadas en el fenómeno que se estudia, en un intento por medir el nivel de conocimiento que tienen los estudiantes que constituyen la población de estudio en esta investigación, siendo estas: Educación Financiera como variable independiente, la cual integra las dimensiones ingreso, administración del dinero, ahorro e inversión y gasto y crédito. Y como variable dependiente es la Inclusión financiera.

De ahí que, tomando de referencia el modelo de Mandell (2008) y el de Child and Youth se establece el modelo teorico causal mostrado en la figura 1:

Figura 1.

Modelo teórico causal de Estudio (constructo)

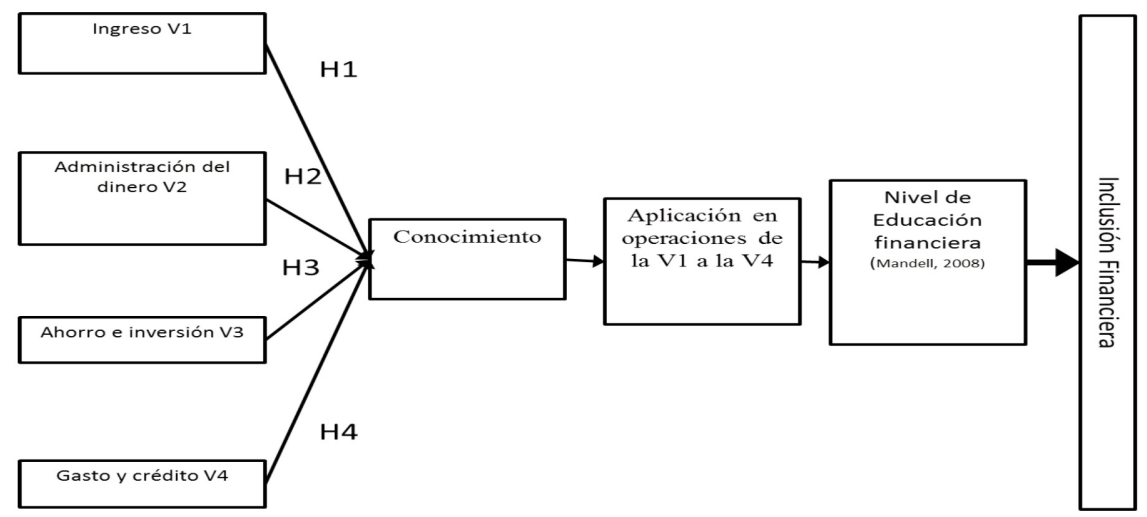

Lo anterior permite establecer como hipótesis de investigación:

Hi: Los estudiantes de educación media superior poseen conocimiento en tópicos financieros, específicamente de administración de dinero, ahorro-inversión y finalmente en gasto-crédito.

Al respecto del contexto mexicano podemos señalar, que la región sureste del pais es la zona que presenta el más lento crecimiento de los índices de acceso y uso de los servicios financieros del país (CNBV, 2012). De ahí que se toma al estado de Veracruz para realizar esta investigación, donde existen 41 bachilleratos de educación tecnológica. De aquí, resulta interesante observar que 
saben los jóvenes que están próximos a iniciar su edad financieramente adulta, acerca de los conceptos que se utilizan en el lenguaje de los productos y servicios financieros, lo cual empata con el estudio realizado por Mandell (2008) en jóvenes de bachillerato en Estados Unidos.

El estudio se lleva a cabo en 3 municipios del estado, distribuido en zona norte, centro y sur, focalizando el estudio en 6 planteles con una población total de 7,668 estudiantes.

Muestra:

Para calcular la muestra se sigue el procedimiento que señala Levin (2002) citado por MorenoGarcía, García-Santillán y Munguía-Tiburcio (2013), el cual sugiere utilizar la siguiente fórmula:

$$
n=\frac{N Z^{2}(P)(Q)}{e^{2}(N-1)+Z^{2}(p q)}
$$

Donde:

$\mathrm{N}=7,936$ población (alumnos inscritos)

$n=$ muestra

$e=$ error permitido $(0.05)$

$Z=$ nivel de confiabilidad (1.96)

$\mathrm{p}=$ probabilidad del evento a favor $(0.5)$

$q=$ probabilidad del evento en contra $(0.5)$

Resolviendo tenemos que:

$$
\begin{aligned}
& n=\frac{N * Z^{2}(P)(Q)}{e^{2}(N-1)+Z^{2}(P)(Q)}=\frac{7,936 * 1.96^{2}(0.5)(0.5)}{.05^{2}(7,936-1)+1.96^{2}(.5)(.5)} \\
& n=\frac{7,936 * 3.8416(0.25)}{.0025(7,935)+3.8416(.25)}=\frac{7,621.7344}{19.8375+0.9604}=366.466537 \\
& n=367
\end{aligned}
$$

Lo que nos arroja un resultado de 367 alumnos a encuestar, distribuidos de la siguiente manera (tabla 1): 
Tabla 1.

Estratificación por municipio y plantel

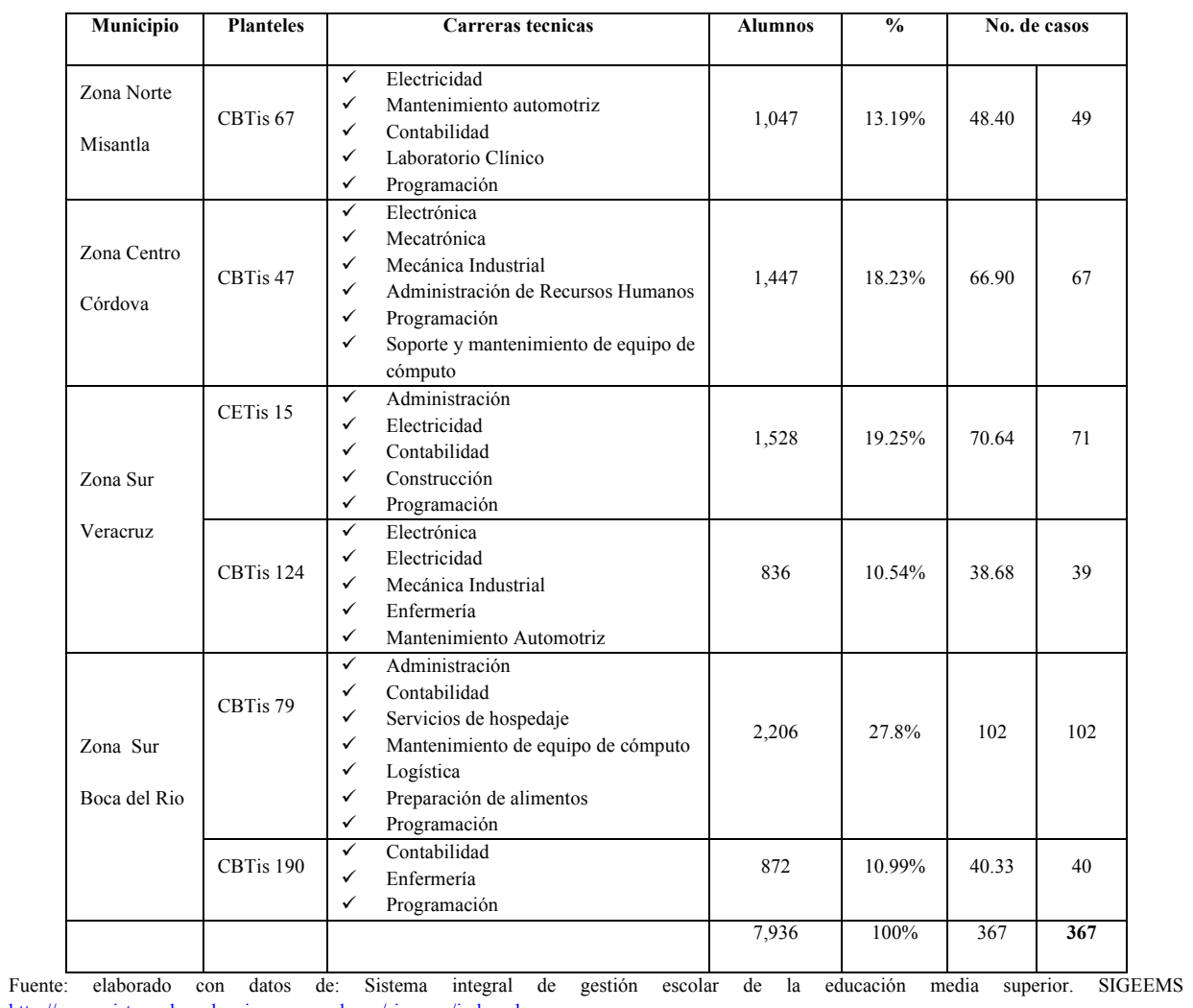

http://www.sistemadeevaluacion.sems.gob.mx/sigeems/index.php

\section{INSTRUMENTO}

A partir del instrumento diseñado por Contreras-Rodríguez (2016), denominado EDUFINANMS $^{4}$, mismo que tiene la finalidad de recabar información relacionada al conocimiento que en tópicos financieros poseen los estudiantes de último año de educación media superior. Consta de 2 partes: el perfil sociodemográfico del encuestado y la sección $\mathrm{A}$, en la cual se tienen 31 indicadores sobre el conocimiento que tienen los jóvenes de las variables de administración de dinero, ahorro e inversión, así como de gasto y crédito. Los ítems están estructurados de acuerdo al método de casos, es decir, cada pregunta presenta al alumno un mini-caso simulando una situación relacionada a rubros financieros con la que se enfrentarían en la vida real en la edad adulta.

\section{III.1 Validación del instrumento}

Se realiza la validación del instrumento, obteniendo lo siguiente: 


\begin{tabular}{|c|c|c|c|c|c|}
\hline \multicolumn{6}{|c|}{$\begin{array}{c}\text { Resumen de la escala: Media=226.022 Std.Dv. }=20.7502 \text { Casos validados: } 368 \\
\text { Alfa de Cronbach: } .788120 \text { Alfa estandarizada: } .780780\end{array}$} \\
\hline & Mean if & Var. if & StDv. if & Itm-Totl & Alpha if \\
\hline Género & 224.4375 & 430.6265 & 20.75154 & -0.071754 & 0.789491 \\
\hline EDAD & 223.3206 & 428.6635 & 20.70419 & 0.014788 & 0.788756 \\
\hline EDO CIVIL & 221.1223 & 429.2595 & 20.71858 & -0.008199 & 0.789006 \\
\hline EST. LABORAL & 222.9919 & 429.1440 & 20.71579 & -0.001892 & 0.788851 \\
\hline INGRESO & 222.4294 & 415.6635 & 20.38783 & 0.131376 & 0.789565 \\
\hline ATN. MÉDICA & 222.3125 & 412.2692 & 20.30441 & 0.091733 & 0.796506 \\
\hline PARENTESCO CON JEFE HOGAR & 223.8913 & 428.9773 & 20.71177 & 0.007438 & 0.788681 \\
\hline INGREUA01 & 222.8831 & 424.7934 & 20.61052 & 0.093831 & 0.788004 \\
\hline INGREUA02 & 223.0136 & 427.9047 & 20.68586 & 0.049250 & 0.788265 \\
\hline INGREUA03 & 223.0299 & 425.1213 & 20.61847 & 0.126617 & 0.787239 \\
\hline INGREUA04 & 223.9022 & 430.0665 & 20.73804 & -0.042304 & 0.789348 \\
\hline INGREUA05 & 224.2473 & 427.3438 & 20.67229 & 0.033772 & 0.789099 \\
\hline INGREUA06 & 223.3206 & 419.3863 & 20.47892 & 0.196049 & 0.785791 \\
\hline INGREUA07 & 224.5978 & 431.2948 & 20.76764 & -0.075151 & 0.790865 \\
\hline ADMIDICO01 & 224.1005 & 429.6991 & 20.72919 & -0.026332 & 0.789079 \\
\hline ADMIDICO02 & 223.4348 & 429.2567 & 20.71851 & -0.023093 & 0.791007 \\
\hline ADMIDICO03 & 224.0081 & 426.7418 & 20.65773 & 0.026610 & 0.790129 \\
\hline ADMIDICO04 & 223.5408 & 424.8842 & 20.61272 & 0.068005 & 0.788999 \\
\hline ADMIDICO05 & 224.0897 & 427.9621 & 20.68724 & 0.017398 & 0.789405 \\
\hline AHOINCA01 & 223.8288 & 432.0278 & 20.78528 & -0.094033 & 0.791394 \\
\hline AHOINCA02 & 223.3315 & 422.0096 & 20.54287 & 0.099920 & 0.788724 \\
\hline AHOINCA03 & 222.3723 & 421.8532 & 20.53907 & 0.193775 & 0.785995 \\
\hline AHOINCA04 & 222.6060 & 424.7062 & 20.60840 & 0.093277 & 0.788045 \\
\hline AHOINCA05 & 223.3505 & 426.2603 & 20.64607 & 0.032689 & 0.790162 \\
\hline AHOINCA06 & 224.1331 & 429.4089 & 20.72218 & -0.024768 & 0.790735 \\
\hline AHOINCA07 & 223.8261 & 424.9426 & 20.61414 & 0.076568 & 0.788576 \\
\hline AHOINCA08 & 223.4538 & 423.3185 & 20.57471 & 0.105699 & 0.787999 \\
\hline GASCRECA01 & 223.4294 & 430.0005 & 20.73645 & -0.043379 & 0.792942 \\
\hline GASCRECA02 & 223.8288 & 425.4897 & 20.62740 & 0.085456 & 0.788072 \\
\hline GASCRECA03 & 223.3777 & 428.7785 & 20.70697 & -0.001674 & 0.789557 \\
\hline GASCRECA04 & 223.0951 & 422.3524 & 20.55121 & 0.121827 & 0.787656 \\
\hline GASCRECA05 & 223.6087 & 418.1295 & 20.44822 & 0.200520 & 0.785672 \\
\hline GASCRECA06 & 223.4348 & 427.3762 & 20.67308 & 0.018745 & 0.790012 \\
\hline GASCRECA07 & 223.1413 & 423.8496 & 20.58761 & 0.091443 & 0.788390 \\
\hline GASCRECA08 & 223.5951 & 434.7247 & 20.85005 & -0.131782 & 0.794721 \\
\hline GASCRECA09 & 223.6223 & 426.0992 & 20.64217 & 0.034107 & 0.790213 \\
\hline GASCRECA10 & 223.3967 & 423.1796 & 20.57133 & 0.079475 & 0.789305 \\
\hline GASCRECA11 & 223.5761 & 422.7279 & 20.56035 & 0.165253 & 0.786527 \\
\hline GASCRECO01 & 222.6902 & 415.9420 & 20.39466 & 0.202077 & 0.785717 \\
\hline GASCRECO02 & 222.3397 & 412.3819 & 20.30719 & 0.314799 & 0.782583 \\
\hline GASCRECO03 & 222.3859 & 409.5685 & 20.23780 & 0.378159 & 0.780907 \\
\hline GASCRECO04 & 222.3315 & 408.2162 & 20.20436 & 0.434549 & 0.779738 \\
\hline GASCRECO05 & 222.3505 & 410.2766 & 20.25529 & 0.341863 & 0.781699 \\
\hline GASCRECO06 & 222.3940 & 411.8746 & 20.29469 & 0.308581 & 0.782633 \\
\hline GASCREUA01 & 221.9592 & 407.8435 & 20.19514 & 0.414347 & 0.779922 \\
\hline GASCREUA02 & 222.8750 & 413.5767 & 20.33659 & 0.252314 & 0.784174 \\
\hline GASCREUA03 & 223.1277 & 414.2527 & 20.35320 & 0.214598 & 0.785393 \\
\hline GASCREUA04 & 223.1522 & 412.0584 & 20.29922 & 0.240010 & 0.784581 \\
\hline GASCREUA05 & 222.2935 & 408.2617 & 20.20549 & 0.353422 & 0.781116 \\
\hline
\end{tabular}


Los resultados obtenidos del alfa de Chronbach (alfa normal y estandarizada) dejan ver que los valores son bajos, si bien no son inferiores a 0.5, tampoco son los que Hair, Anderson, Tatham y Black (1999) señalan como deseables (> .8), de la tabla 9 se puede observar que la fiabilidad de cada uno de los ítems es superior a 0.70, por lo que se puede inferir que tan bien están explicados y entendidos cada uno de los ítems.

\section{ANÁLISIS DE DATOS}

A continuación los resultados que arrojaron el análisis descriptivo correspondiente al perfil sociodemográfico de los encuestados.

\section{1 Análisis descriptivo}

A continuación se presenta el análisis descriptivo de datos del perfil del encuestado.

Tabla 1.

Descriptivos de la variable Género

\begin{tabular}{lccccccc}
\hline \multicolumn{1}{c}{ Plantel } & Variable & $>$ Frecuencia & $\mu$ & D.S. & $\mathrm{P}<0.01$ & SW-W & Lilliefors \\
\hline Zona Norte & & $82 \%$ mujeres & 1.8163 & 0.3912 & $\mathrm{P}=0.00$ & 0.4716 & $\mathrm{P}<0.01$ \\
Zona Centro & \multirow{2}{*}{ Género } & $72 \%$ mujeres & 1.7164 & 0.4541 & $\mathrm{P}=0.00$ & 0.5646 & $\mathrm{P}<0.01$ \\
Zona Sur & & $50 \%$ Hombres & 1.504 & 0.501 & $\mathrm{P}=0.00$ & 0.6364 & $\mathrm{P}<0.01$ \\
\hline
\end{tabular}

Fuente: Elaboración propia

En la tabla 1 relativa a la variable género, se puede apreciar que el género mujer, presenta mayor frecuencia en los planteles de la Zona Norte y Zona Centro ( $82 \%$ y $72 \%$ ) adicionalmente en las instituciones seleccionadas de la Zona Sur el rango de mujer y hombre representan un $50 \%$ cada uno. Las pruebas de bondad de ajuste de Kolmogorov -Smirnov con la corrección de Lilliefors cuyo resultado es $<0.01$, apoya esta medición y se puede decir que los datos proceden de una distribución normal.

Tabla 2.

Descriptivos de la variable Edad

\begin{tabular}{|c|c|c|c|c|c|c|c|}
\hline Plantel & Variable & $>$ Frecuencia & $\mu$ & D.s. & $\mathrm{P}<0.01$ & SW-W & Lilliefors \\
\hline Zona Norte & & $\begin{array}{c}53 \% 18 \\
\text { cumplidos }\end{array}$ & 2.6122 & 0.5707 & $\begin{array}{c}\mathrm{P}= \\
0.00\end{array}$ & 0.726 & $\mathrm{P}<0.01$ \\
\hline Zona Centro & Edad & $\begin{array}{c}57 \% 18 \\
\text { cumplidos }\end{array}$ & 2.6119 & 0.5762 & $\mathrm{P}=0.00$ & 0.7471 & $\mathrm{P}<0.01$ \\
\hline Zona Sur & & $\begin{array}{c}56 \% 18 \\
\text { cumplidos }\end{array}$ & 2.7421 & 0.6192 & $\mathrm{P}=0.00$ & 0.7658 & $\mathrm{P}<0.01$ \\
\hline
\end{tabular}

Fuente: Elaboración propia

En relación a la variable edad, la tabla 2 muestra que la edad de 18 años cumplidos, presenta mayor frecuencia en las 6 instituciones de la población estudiada: en la Zona Norte (53\%), Centro $(57 \%)$ y los planteles de la Zona Sur (56\%). Las pruebas de bondad de ajuste de Kolmogorov -Smirnov con la corrección de Lilliefors cuyo resultado es $<0.01$, apoya esta medición y se puede decir que los datos proceden de una distribución normal. 
Tabla 3.

Descriptivos de la variable Estado civil

\begin{tabular}{|c|c|c|c|c|c|c|c|}
\hline Plantel & Variable & $>$ Frecuencia & $\mu$ & D.s. & $\mathrm{P}<0.01$ & SW-W & Lilliefors \\
\hline Zona Norte & Estado & $\begin{array}{l}100 \% \\
\text { Soltero }\end{array}$ & N/A & N/A & N/A & N/A & N/A \\
\hline Zona Centro & civil & $93 \%$ Soltero & 4.791 & 0.8445 & $\mathrm{P}=0.00$ & 0.2587 & $\mathrm{P}<0.01$ \\
\hline Zona Sur & & $97 \%$ Soltero & 4.9087 & 0.5535 & $\mathrm{P}=0.00$ & 0.1525 & $\mathrm{P}<0.01$ \\
\hline
\end{tabular}

Fuente: Elaboración propia

Para el análisis de la variable Estado civil, en la tabla 3, se puede percibir que el estado civil Soltero, presenta mayor frecuencia en toda la población, específicamente en la Zona Norte es del $100 \%$. De manera similar, en la selección de la Zona Centro se observa un $93 \%$ y en la Zona Sur un $97 \%$, por lo que se desprende que el rango mayor es soltero. Las pruebas de bondad de ajuste de Kolmogorov -Smirnov con la corrección de Lilliefors cuyo resultado es $<0.01$, apoya esta medición y se puede decir que los datos proceden de una distribución normal, exceptuando las instituciones de la Zona Norte donde no aplican la pruebas de bondad.

Tabla 4.

Descriptivos de la variable Estatus Laboral

\begin{tabular}{cccccccc}
\hline \multicolumn{1}{c}{ Plantel } & Variable & $>$ Frecuencia & $\mu$ & D.s. & $\mathrm{P}<0.01$ & SW-W & Lilliefors \\
\hline Zona Norte & & $\begin{array}{c}84 \% \text { Solo } \\
\text { estudia }\end{array}$ & 3.0612 & 0.4747 & $\mathrm{P}=0.00$ & 0.531 & $\mathrm{P}<0.01$ \\
Zona Centro & $\begin{array}{c}\text { Estatus } \\
\text { Laboral }\end{array}$ & $\begin{array}{c}75 \% \text { Solo } \\
\text { estudia }\end{array}$ & 2.9851 & 0.5901 & $\mathrm{P}=0.00$ & 0.709 & $\mathrm{P}<0.01$ \\
Zona Sur & $\begin{array}{c}75 \% \text { Solo } \\
\text { estudia }\end{array}$ & 3.0357 & 0.5527 & $\mathrm{P}=0.00$ & 0.6697 & $\mathrm{P}<0.01$ \\
\hline
\end{tabular}

Fuente: Elaboración propia

La tabla 4 menciona la frecuencia para la variable de Estatus Laboral, habiendo mayor frecuencia de la población que solo estudia, en el total de las instituciones analizadas. Diferenciado por zonas, en la Zona Norte (84\%), Centro (75\%) y los planteles de la Zona Sur (75\%). Las pruebas de bondad de ajuste de Kolmogorov -Smirnov con la corrección de Lilliefors cuyo resultado es $<0.01$, sustentan esta medición de ahí que se puede indicar que los datos proceden de una distribución normal.

Tabla 5 .

Descriptivos de la variable Ingreso

\begin{tabular}{lccccccc}
\hline \multicolumn{1}{c}{ Plantel } & Variable & $>$ Frecuencia & $\mu$ & D.s. & $\mathrm{P}<0.01$ & SW-W & Lilliefors \\
\hline Zona Norte & $\begin{array}{c}67 \% \text { No } \\
\text { aplica }\end{array}$ & 3.6939 & 1.8952 & $\mathrm{P}=0.00$ & 0.5916 & $\mathrm{P}<0.01$ \\
Zona Centro & Ingreso & $\begin{array}{c}82 \% \text { No } \\
\text { aplica } \\
58 \% \text { No } \\
\text { aplica }\end{array}$ & 4.3284 & 1.4605 & $\mathrm{P}=0.00$ & 0.4772 & $\mathrm{P}<0.01$ \\
Zona Sur & & & 1.9415 & $\mathrm{P}=0.00$ & 0.6429 & $\mathrm{P}<0.01$ \\
\hline Fuente: Elaboración propia & & & & & &
\end{tabular}

Fuente: Elaboración propia 
La tabla 5 se muestra la frecuencia para la variable de Estatus Laboral, en donde se denota mayor frecuencia en la población que solo estudia, en las seis instituciones analizadas: en la Zona Norte (84\%), Centro (75\%) y los planteles de la Zona Sur (58\%). Las pruebas de bondad de ajuste de Kolmogorov -Smirnov con la corrección de Lilliefors cuyo resultado es $<0.01$, sustentan esta medición de ahí que se puede indicar que los datos proceden de una distribución normal.

Tabla 6.

Descriptivos de la variable Atención medica

\begin{tabular}{|c|c|c|c|c|c|c|c|}
\hline Plantel & Variable & $>$ Frecuencia & $\mu$ & D.s. & $\mathrm{P}<0.01$ & SW-W & Lilliefors \\
\hline Zona Norte & Atención & $\begin{array}{c}33 \% \text { Seguro } \\
\text { Popular }\end{array}$ & 3.3878 & 2.7372 & $\mathrm{P}=0.00$ & 0.7548 & $\mathrm{P}<0.01$ \\
\hline Zona Centro & medica & $40 \%$ IMSS & 4.6567 & 3.0229 & $\mathrm{P}=0.00$ & 0.7245 & $\mathrm{P}<0.01$ \\
\hline Zona Sur & & $47 \%$ IMSS & 3.5198 & 2.5239 & $\mathrm{P}=0.00$ & 0.7493 & $\mathrm{P}<0.01$ \\
\hline
\end{tabular}

Fuente: Elaboración propia

Mencionando la frecuencia para la variable de Atención médica, la tabla 6 indica mayor frecuencia de individuos que cuentan con Seguro popular en los planteles de la zona Norte (33\%), en cambio en las instituciones de la zona Centro y Sur la mayor frecuencia se presenta en población que cuentan con IMSS (desde un 40 al 47\%). Las pruebas de bondad de ajuste de Kolmogorov -Smirnov con la corrección de Lilliefors cuyo resultado es $<0.01$, sustentan esta medición de ahí que se puede indicar que los datos proceden de una distribución normal.

Tabla 7.

Descriptivos de la variable Parentesco con jefe de familia

\begin{tabular}{lccccccc}
\hline \multicolumn{1}{c}{ Plantel } & Variable & $>$ Frecuencia & $\mu$ & D.s. & $\mathrm{P}<0.01$ & SW-W & Lilliefors \\
\hline Zona Norte & Parentesco & $88 \%$ Hijo & 2.2449 & 0.6931 & $\mathrm{P}=0.00$ & 0.3969 & $\mathrm{P}<0.01$ \\
Zona Centro & $\begin{array}{c}\text { con jefe } \\
\text { de familia }\end{array}$ & $87 \%$ Hijo & 2.1493 & 0.6094 & $\mathrm{P}=0.00$ & 0.4544 & $\mathrm{P}<0.01$ \\
Zona Sur & & $92 \%$ Hijo & 2.1032 & 0.4433 & $\mathrm{P}=0.00$ & 0.317 & $\mathrm{P}<0.01$
\end{tabular}

Fuente: Elaboración propia

Para el análisis de la variable Parentesco con el jefe de familia, en la tabla 7, se puede percibir que en el total de las instituciones de la muestra, se presentan mayor frecuencia la respuesta Hijo; en la Zona Sur es del 92\%, en la Zona Centro (87\%), por lo que se puede afirmar que el rango mayor guarda una relación de Hijo con el jefe de familia. Las pruebas de bondad de ajuste de Kolmogorov -Smirnov con la corrección de Lilliefors cuyo resultado es $<0.01$, apoya esta medición y se puede decir que los datos proceden de una distribución normal.

\section{2 Resultados para la Sección A. Conocimiento Financiero}

Para la interpretación de los resultados correspondiente a la sección A denominada Conocimiento Financiero, se realiza un análisis descriptivo de cada una de las variables distribuidas por zona, para observar el comparativo entre las mismas y con la finalidad de dar respuesta las interrogantes del constructo de Educación Financiera. De ahí que, respecto a la variable Ingreso, que comprende los siguientes ítems del instrumento, se obtuvieron los resultados descritos en la tabla 8: 
Tabla 8.

Frecuencias de respuestas positivas versus negativas, variable Ingreso

\begin{tabular}{|c|c|c|c|c|}
\hline VARIABLE & ÍTEM & ZONA & \% CORRECTO & \% INCORRECTO \\
\hline \multirow{21}{*}{ INGRESO } & \multirow{3}{*}{1} & Z-1 & $55 \%$ & $44 \%$ \\
\hline & & Z-2 & $39 \%$ & $61 \%$ \\
\hline & & Z-3 & $42 \%$ & $58 \%$ \\
\hline & \multirow{3}{*}{2} & Z-1 & $84 \%$ & $16 \%$ \\
\hline & & Z-2 & $82 \%$ & $17 \%$ \\
\hline & & Z-3 & $74 \%$ & $26 \%$ \\
\hline & \multirow{3}{*}{3} & Z-1 & $73 \%$ & $26 \%$ \\
\hline & & Z-2 & $75 \%$ & $25 \%$ \\
\hline & & Z-3 & $67 \%$ & $33 \%$ \\
\hline & \multirow{3}{*}{4} & Z-1 & $69 \%$ & $30 \%$ \\
\hline & & $\mathrm{Z}-2$ & $72 \%$ & $28 \%$ \\
\hline & & Z-3 & $75 \%$ & $24 \%$ \\
\hline & \multirow{3}{*}{5} & Z-1 & $47 \%$ & $53 \%$ \\
\hline & & Z-2 & $48 \%$ & $51 \%$ \\
\hline & & Z-3 & $45 \%$ & $56 \%$ \\
\hline & \multirow{3}{*}{6} & Z-1 & $35 \%$ & $65 \%$ \\
\hline & & Z-2 & $55 \%$ & $44 \%$ \\
\hline & & Z-3 & $19 \%$ & $81 \%$ \\
\hline & \multirow{3}{*}{7} & Z-1 & $6 \%$ & $94 \%$ \\
\hline & & $\mathrm{Z}-2$ & $3 \%$ & $97 \%$ \\
\hline & & Z-3 & $4 \%$ & $96 \%$ \\
\hline
\end{tabular}

Fuente: Elaboración propia

Su representación gráfica es la siguiente:

Gráfica 1.

Descripción de las respuestas correctas e incorrectas sobre Ingreso por zonas. (Fuente: Elaboración propia)

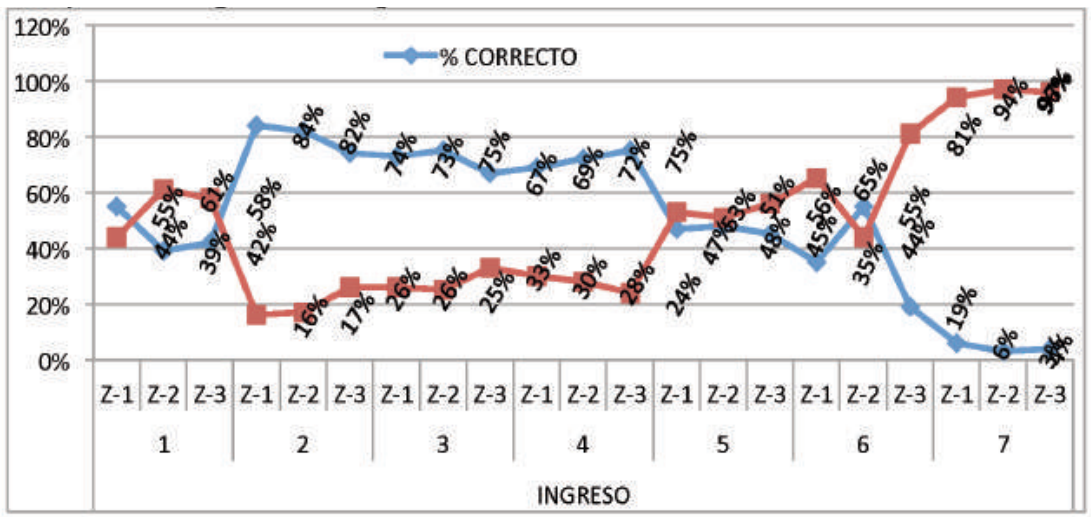


En la gráfica 1, podemos observar cómo los estudiantes de las zonas 2 y 3 , no consideran que un título universitario incrementaría los ingresos que aspiran tener (ítem 1), contrastando este dato, caso contrario que sí consideran que el tomar cursos extracurriculares para mejorar los conocimientos (ítem 2), impacta en un salario a futuro por llegar a ser valioso para su empresa. Además, los alumnos muestran conocimiento en relación a las alternativas de ingreso que pudieran tener en su etapa adulta, o mejor dicho, en la etapa en la cual se insertan al campo laboral formal (ítem 4). Caso contrario en materia de impuestos, el resultado nos lleva a pensar que al parecer los alumnos no logran identificar o precisar con qué tipo de impuesto está gravado el ingreso que percibirán por la jornada laboral (ítem 5). Adicionalmente, tampoco hay evidencia que nos permita decir que el alumno reconoce el efecto de las tasas impositivas en materia fiscal y de seguridad social sobre el ingreso (ítem 7).

En relación a la variable Administración de dinero, se obtuvo para cada uno de los ítems (del 8 al 12) las siguientes frecuencias.

Tabla 9 .

Frecuencias de respuestas positivas versus negativas de la variable Administración de dinero

\begin{tabular}{|c|c|c|c|c|}
\hline VARIABLE & ITEM & ZONA & \% CORRECTO & \% INCORRECTO \\
\hline & & Z-1 & $92 \%$ & $8 \%$ \\
\hline & 8 & $\mathrm{Z}-2$ & $58 \%$ & $42 \%$ \\
\hline & & $Z-3$ & $84 \%$ & $16 \%$ \\
\hline & & Z-1 & $4 \%$ & $96 \%$ \\
\hline & 9 & $\mathrm{Z}-2$ & $4 \%$ & $95 \%$ \\
\hline & & $Z-3$ & $6 \%$ & $93 \%$ \\
\hline & & Z-1 & $33 \%$ & $67 \%$ \\
\hline \multirow{8}{*}{$\begin{array}{c}\text { ADMON } \\
\text { DE DINERO }\end{array}$} & 10 & $\mathrm{Z}-2$ & $19 \%$ & $80 \%$ \\
\hline & & $Z-3$ & $23 \%$ & $77 \%$ \\
\hline & & $\mathrm{Z}-1$ & $12 \%$ & $88 \%$ \\
\hline & 11 & $\mathrm{Z}-2$ & $39 \%$ & $61 \%$ \\
\hline & & $\mathrm{Z}-3$ & $21 \%$ & $80 \%$ \\
\hline & & Z-1 & $29 \%$ & $72 \%$ \\
\hline & 12 & $\mathrm{Z}-2$ & $72 \%$ & $27 \%$ \\
\hline & & Z-3 & $52 \%$ & $48 \%$ \\
\hline
\end{tabular}

Fuente: Elaboración propia 
Cuya representación gráfica se muestra a continuación:

Gráfica 2.

Descripción de las respuestas correctas e incorrectas variable Administración de dinero.

(Fuente: Elaboración propia)

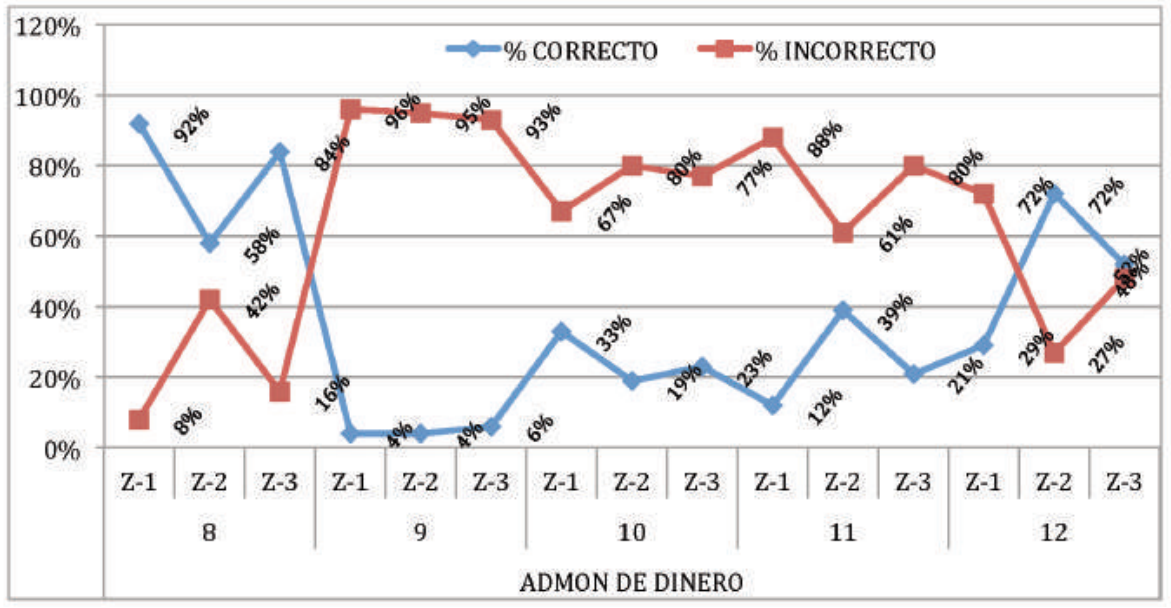

En cuanto a la variable administración de dinero, en la gráfica número 2, podemos inferir que si bien es cierto que los alumnos de las 3 zonas estudiadas, conocen de la existencia de las pensiones y en general su definición (ítem 8), por el contrario, a partir del mismo resultado se puede contrastar que en temas de seguros no poseen conocimiento alguno, específicamente si se refiere a seguros de vida 0 como el seguro de automóvil (ítems 10 y 11), en el mismo sentido si hablamos de inflación y el impacto que esta tiene en las finanzas personales (ítem 9). Caso contrario, sí conocen el canal por el cual ellos poseen servicios de salud, en otras palabras, están conscientes de contar con un servicio medico a través de sus padres (ítem 12), y el riesgo que corre su cobertura si sus padres se quedan sin empleo.

La variable Ahorro e inversión, se obtuvo para cada uno de los ítems (del 13 al 20) las siguientes frecuencias. 
NIVEL DE CONOCIMIENTO QUE TIENEN LOS ALUMNOS DE NIVEL MEDIO SUPERIOR EN TÓPICOS FINANCIEROS SOBRE GASTO Y CRÉDITO, AHORRO E INVERSIÓN Y LA ADMINISTRACIÓN DEL DINERO

Tabla 10.

Frecuencias de respuestas positivas versus negativas variable Ahorro e inversión

\begin{tabular}{|c|c|c|c|c|}
\hline VARIABLE & ITEM & ZONA & \% CORRECTO & \% INCORRECTO \\
\hline \multirow{24}{*}{ AHORRO E INVERSIÓN } & \multirow{3}{*}{13} & $\mathrm{Z}-1$ & $65 \%$ & $34 \%$ \\
\hline & & $\mathrm{Z}-2$ & $69 \%$ & $30 \%$ \\
\hline & & Z-3 & $60 \%$ & $41 \%$ \\
\hline & \multirow{3}{*}{14} & $\mathrm{Z}-1$ & $59 \%$ & $41 \%$ \\
\hline & & $\mathrm{Z}-2$ & $63 \%$ & $36 \%$ \\
\hline & & $\mathrm{Z}-3$ & $43 \%$ & $57 \%$ \\
\hline & \multirow{3}{*}{15} & $\mathrm{Z}-1$ & $98 \%$ & $2 \%$ \\
\hline & & $\mathrm{Z}-2$ & $90 \%$ & $10 \%$ \\
\hline & & $\mathrm{Z}-3$ & $80 \%$ & $21 \%$ \\
\hline & \multirow{3}{*}{16} & $\mathrm{Z}-1$ & $24 \%$ & $75 \%$ \\
\hline & & $\mathrm{Z}-2$ & $12 \%$ & $87 \%$ \\
\hline & & $\mathrm{Z}-3$ & $20 \%$ & $81 \%$ \\
\hline & \multirow{3}{*}{17} & Z-1 & $33 \%$ & $68 \%$ \\
\hline & & $\mathrm{Z}-2$ & $16 \%$ & $84 \%$ \\
\hline & & Z-3 & $29 \%$ & $71 \%$ \\
\hline & \multirow{3}{*}{18} & $\mathrm{Z}-1$ & $12 \%$ & $88 \%$ \\
\hline & & $\mathrm{Z}-2$ & $9 \%$ & $91 \%$ \\
\hline & & $\mathrm{Z}-3$ & $10 \%$ & $90 \%$ \\
\hline & \multirow{3}{*}{19} & Z-1 & $18 \%$ & $82 \%$ \\
\hline & & $\mathrm{Z}-2$ & $19 \%$ & $80 \%$ \\
\hline & & Z-3 & $18 \%$ & $81 \%$ \\
\hline & \multirow{3}{*}{20} & $\mathrm{Z}-1$ & $16 \%$ & $83 \%$ \\
\hline & & $\mathrm{Z}-2$ & $34 \%$ & $65 \%$ \\
\hline & & Z-3 & $23 \%$ & $77 \%$ \\
\hline
\end{tabular}

Fuente: Elaboración propia

Graficando las frecuencias anteriores de la siguiente manera: 
Gráfica 3.

Descripción de las respuestas correctas e incorrectas correspondientes a la variable Ahorro e Inversión. (Fuente: Elaboración propia)

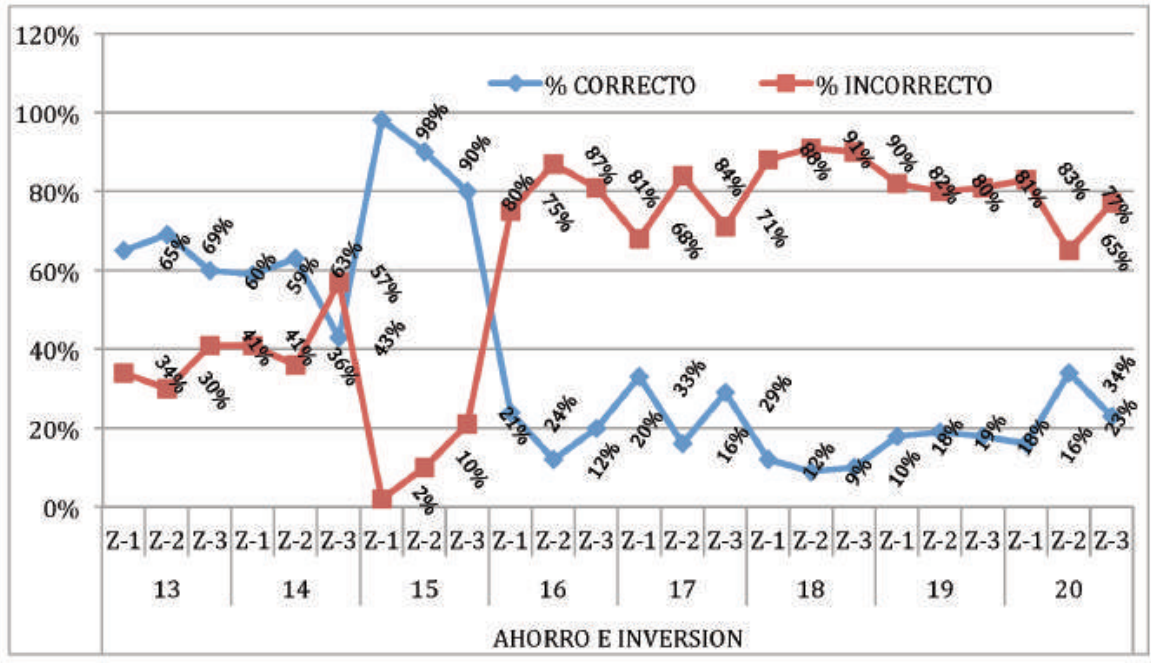

Conforme a la gráfica 3, podemos notar a partir del análisis de los datos, que los estudiantes de las zonas estudiadas, manifiestan tener un conocimiento elevado de la utilidad de realizar un ahorro (ítem 13), como consecuencia conocen algunos beneficios que trae consigo un ahorro, de ello resulta necesario afirmar que también identifican algunos canales básicos de ahorros, como una cuenta bancaria por ejemplo (ítem15).

Contrastando este dato, se denota falta de conocimiento hacia los instrumentos de inversión más complejos (ítem 16), a saber, aquellos instrumentos que por su naturaleza, están asociados a los mercados de capitales, siendo el caso más específico, aquellos que se negocian en la Bolsa de Valores. El resultado de esto último, nos lleva a pensar que al parecer los alumnos no cuentan con un conocimiento hacia estos instrumentos. Adicionalmente los resultados tampoco arrojan evidencia que nos permita decir que los alumnos conocen los bonos de la Tesorería (ítem 18). Si bien aun no tienen contacto con temas financieros tan complejos, tampoco han tenido ningún contacto con terceras personas que conozcan de estos conceptos, según parece.

Finalmente, la variable Gasto y crédito queda constituido por las siguientes frecuencias para cada uno de los ítems (del 21 al 31): 
NIVEL DE CONOCIMIENTO QUE TIENEN LOS ALUMNOS DE NIVEL MEDIO SUPERIOR EN TÓPICOS FINANCIEROS SOBRE GASTO Y CRÉDITO, AHORRO E INVERSIÓN Y LA ADMINISTRACIÓN DEL DINERO

Tabla 11.

Frecuencias de respuestas positivas versus negativas variable Gasto y crédito

\begin{tabular}{|c|c|c|c|c|}
\hline VARIABLE & ITEM & ZONA & \% CORRECTO & \% INCORRECTO \\
\hline & & Z-1 & $41 \%$ & $59 \%$ \\
\hline & 21 & $\mathrm{Z}-2$ & $45 \%$ & $54 \%$ \\
\hline & & Z-3 & $33 \%$ & $66 \%$ \\
\hline & & Z-1 & $59 \%$ & $40 \%$ \\
\hline & 22 & $\mathrm{Z}-2$ & $60 \%$ & $39 \%$ \\
\hline & & $Z-3$ & $58 \%$ & $42 \%$ \\
\hline & & Z-1 & $49 \%$ & $51 \%$ \\
\hline & 23 & $\mathrm{Z}-2$ & $46 \%$ & $54 \%$ \\
\hline & & Z-3 & $49 \%$ & $51 \%$ \\
\hline & & Z-1 & $59 \%$ & $40 \%$ \\
\hline & 24 & $\mathrm{Z}-2$ & $55 \%$ & $44 \%$ \\
\hline & & Z-3 & $38 \%$ & $62 \%$ \\
\hline & & Z-1 & $37 \%$ & $64 \%$ \\
\hline & 25 & $\mathrm{Z}-2$ & $28 \%$ & $71 \%$ \\
\hline & & Z-3 & $30 \%$ & $69 \%$ \\
\hline & & Z-1 & $14 \%$ & $86 \%$ \\
\hline \multirow[t]{17}{*}{ GASTO Y CREDITO } & 26 & $\mathrm{Z}-2$ & $31 \%$ & $68 \%$ \\
\hline & & Z-3 & $25 \%$ & $75 \%$ \\
\hline & & Z-1 & $55 \%$ & $44 \%$ \\
\hline & 27 & $\mathrm{Z}-2$ & $48 \%$ & $52 \%$ \\
\hline & & Z-3 & $42 \%$ & $57 \%$ \\
\hline & & Z-1 & $47 \%$ & $53 \%$ \\
\hline & 28 & $\mathrm{Z}-2$ & $46 \%$ & $53 \%$ \\
\hline & & Z-3 & $33 \%$ & $67 \%$ \\
\hline & & $\mathrm{Z}-1$ & $51 \%$ & $49 \%$ \\
\hline & 29 & $\mathrm{Z}-2$ & $45 \%$ & $54 \%$ \\
\hline & & Z-3 & $35 \%$ & $65 \%$ \\
\hline & & Z-1 & $41 \%$ & $59 \%$ \\
\hline & 30 & Z-2 & $39 \%$ & $61 \%$ \\
\hline & & Z-3 & $48 \%$ & $51 \%$ \\
\hline & & Z-1 & $55 \%$ & $44 \%$ \\
\hline & 31 & Z-2 & $66 \%$ & $34 \%$ \\
\hline & & Z-3 & $44 \%$ & $56 \%$ \\
\hline
\end{tabular}

Fuente: Elaboración propia

Graficando las frecuencias anteriores de la siguiente manera: 
Gráfica 4.

Descripción de las respuestas correspondientes a la variable Gasto y crédito, positivas versus negativas.

(Fuente: Elaboración propia)

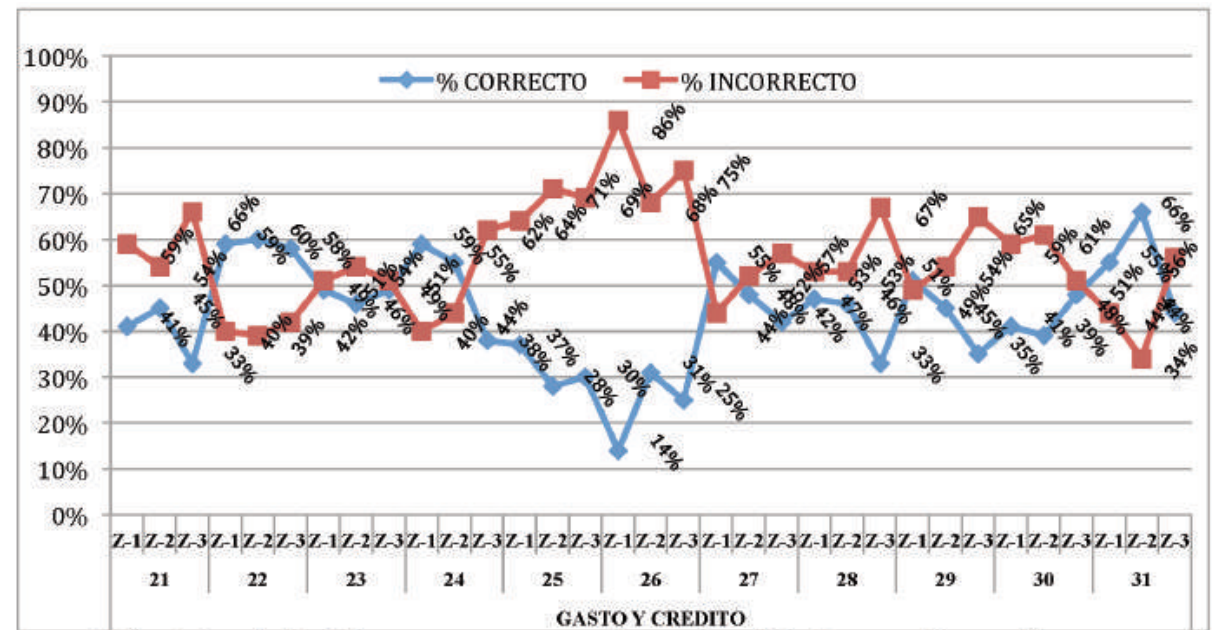

Gráfica 4. Descripción de las respuestas correspondientes a la variable Gasto y crédito, positivas versus negativas. (Fuente: Elaboración propia)

En la gráfica anterior, referente a la variable gasto y crédito los alumnos apuntan a una profunda falta de conocimiento en tópicos relacionados al gasto. En primer lugar se evidencia que no pueden diferenciar cuando se trata de un gasto necesario o innecesario (ítem 21); por otra parte, tampoco demuestran conocer las operaciones crediticias sencillas, por ejemplo, los costos de cargo financiero (ítem 26). No obstante, los alumnos tienen conocimientos en relación a las tarjetas de crédito, las asocian con un gasto (ítem 24), además conocen algunas instituciones que brindan asesoría de crédito responsable, como la CONDUSEF, la cual consideran ellos que representa un servicio de protección a sus derechos (ítem 31).

\section{CONCLUSIONES}

Los resultados que arrojó el análisis realizado a la información obtenida en la aplicación del instrumento en campo, proporcionan información respecto a la educación financiera que prevalece en la población estudiada. Por ello es importante señalar que derivado de estos hallazgos se tendrá un panorama actual sobre el conocimiento en materia financiera que poseen los estudiantes que están próximos a entrar a la edad financieramente adulta, y sobre todo, permite identificar la percepción que tienen acerca de los tópicos financieros.

En el caso de pensiones, el mayor porcentaje para las tres zonas analizadas, conoce de la existencia de las pensiones y su definición en general, contestando correctamente el $92 \%$ en la zona norte y $84 \%$ en la zona sur, en el caso de la zona centro esta proporción disminuye con una mayoría relativa del $58 \%$ que posee conocimiento general de las pensiones (ítem 8). Contrastando lo anterior, la mayoría de los estudiantes para las zonas analizadas contestó de manera incorrecta en el tema de inflación (ítem 9), con puntajes del 96\% (zona norte), 95\% (zona centro) y 93\% (zona sur). Es decir, los estudiantes desconocen el impacto que tiene la inflación en sueldos, salarios y pensiones (ver tabla 9 y gráfica 2). 
Para el tema de seguros (ítems 19 y 11), la mayoría de los estudiantes desconoce lo relativo a seguros de vida y el impacto que tienen en el nivel de salario, pues en la zona norte el $67 \%$, zona centro $80 \%$ y en la zona sur el $77 \%$, contestaron incorrectamente. En este mismo sentido, el $88 \%$ no posee conocimiento vinculado a seguros de automóvil (ver tabla 9). Lo que coincide con Atkinson, McKay, Kempson y Collard (2006), quienes señalan que la carencia de conocimiento de la población en materia financiera, repercute en las decisiones en temas financieros.

En el mismo sentido, Hastings y Tejeda (2008), refieren que las personas con menor conocimiento financiero tienen una menor probabilidad de planificar su jubilación y de elegir fondos de pensión con comisiones más bajas. Así como Mandell (2008), que señala que las decisiones financieras de los consumidores tienen un efecto negativo en la economía, tales como una baja en la tasa de ahorro y la formación de capital, un bajo nivel de ahorro para el retiro, y una tasa más alta de la distribución desigual del ingreso y la riqueza.

Los estudiantes de la muestra analizada, tienen conocimientos en relación a la utilidad de mantener un ahorro, pues el $65 \%$ (zona norte), 69\% (zona centro) y 60\% (zona sur) de los estudiantes contestó de manera correcta en los casos que se les presentaron respecto a las opciones y beneficios del ahorro (ítems 13 y 14). En el mismo sentido, la mayoría señala conocer algunos canales básicos de ahorro (ítem 15), como una cuenta bancaria por ejemplo, pues el 98\%, 90\% y 80\%, contestaron correctamente (ver tabla 11 y gráfica 3 ). El resultado obtenido nos lleva a establecer una postura distinta a Lusardi (2008) quien afirma que a bajos niveles de educación financiera disminuye la capacidad de ahorrar, planificar y asimismo, a una modesta acumulación de riqueza, pues en la población analizada, los estudiantes manifiestan tener un conocimiento elevado de la utilidad de realizar un ahorro.

Aunado a lo anterior, la mayoría (54\%) muestran desconocimiento en relación a la función de los cajeros automáticos (ítem 23). En contraste a lo anterior, aun cuando el 55\% (zona norte), 66\% (centro) y el 56\% (z-sur) conocen de la existencia de instituciones crediticias para la protección y asesoría de los usuarios de los servicios y productos financieros, como la CONDUSEF, la mayoría de los estudiantes desconoce que existe un historial crediticio y la función de éste para las instituciones financieras. El porcentaje de respuestas incorrectas en este sentido va desde un $53 \%$ (zonas norte y centro) hasta $67 \%$ (sur).

Con este trabajo, se obtuvo evidencia empírica que permite explicar cuál es el estado actual que guardan las variables de estudio en su conjunto, por consiguiente, se logra establecer el nivel de educación financiera que poseen los estudiantes de nivel medio superior en el estado de Veracruz y la percepción que tienen acerca de estos temas financieros. De esta forma los hallazgos se suman al conocimiento existente, pero ahora, como una contribución en contextos latinoamericanos, que es el caso que nos ocupa en este estudio.

Pero mas allá de establecer la ausensia de conocimiento por parte de los alumnos, Como se ha comentado anteriormente, los sistemas financieros van ampliando cada vez más la oferta de productos y servicios, por lo tanto, la educación financiera se convierte en una herramienta que coadyuva a que las personas con el conocimiento en el tema, logren tomar decisiones que sean más analíticas para conservar su patrimonio y mejorar en términos económicos, lo relacionado a las finanzas familiares. Esto es relevante, ya que ha sido uno de los factores, -entre otros-, que mal administrado puede llevar a los países a colapsos económicos muy importantes.

Si las familias están mal financieramente hablando, no se da la dinámica que los sistemas financieros requieren para generar esa actividad económica que es necesaria en toda economía.

Lo que en este estudio se propone a partir de los hallazgos, es resaltar la importancia de incluir en la currícula escolar temas que fomentan y ayuden a desarrollar las habilidades y capacidades de 
los estudiantes en relación al manejo de las finanzas personales, para que a través de una metodología de casos, los lleve a una realidad contextualizada en su futuro financiero. Es decir, desde la edad previa a la etapa adulta, que tengan ya un acercamiento hacia las finanzas personales, sus conceptos, instituciones y organismos involucrados, productos y servicios, entre otros factores.

Consideramos que a partir de que el estudiante tenga consciencia de la existencia de estos conceptos financieros, el uso y aplicación de los mismos, tendrá mayores argumentos para lograr tomar las mejores decisiones financieras en su vida. Además, desarrollará su capacidad analítica en temas económicos y todo lo relacionado a las finanzas familiares, en los que estamos inmersos dentro de la sociedad en que vivimos.

Por último, podemos señalar que tener finanzas personales estables, aunque esté latente la limitante de recursos, contribuirá a mantener el bienestar en los hogares, ya que es a consecuencia de las crisis financieras que se detonan problemas sociales en el país.

De ahí que se sugiere realizar estudios en poblaciones de edad adulta con el determinante del nivel académico de bachillerato, es decir, adultos cuyo ultimo nivel de estudios haya sido nivel bachiIlerato, aun cuando tengan edad adulta, a fin de conocer el nivel que actualmente poseen quienes ya se encuentran inmersos en la oferta de servicios y productos financieros.

En este orden de ideas, se sugiere analizar si la oferta digital de servicios y productos financieros está contribuyendo al incremento de la inclusión financiera en sectores de nivel de bajo ingreso.

\section{REFERENCIAS}

Allen, F., Bartiloro, L. y Kowalewski, 0. (2006). The financial system of the EU 25. Munich personal RePEc Archive. Vol. 652.

AFI (2013)._Ubicando la inclusión financiera en el mapa mundial. Informe sobre avances de la Declaración Maya 2013. México: Alliance for Financial Inclusion. http://www.afiglobal.org/sites/default/files/publications/afi 2013 maya progress report sp final.pdf

Atkinson, A., S. McKay, E. Kempson y S. Collard. (2006). Levels of Financial Capability in the UK: Results of a Baseline Survey. Londres: Autoridad de Servicios Financieros. Recuperado de: http://www.pfrc.bris.ac.uk/completed research/Reports/Fincap baseline results_06.pdf

Banco Mundial (2014). Global financial development report. International Bank for Reconstruction and Development, Washington D.C.

BANXIC0. (2013). Palabras del gobernador del banco de México, Dr. Agustín Carstens durante el seminario: Inclusión Financiera responsable y exitosa: modelos internacionales. Mayo 2013 México D.F. Recuperado de: http://www.banxico.org.mx/publicaciones-y-discursos/discursosy-presentaciones/discursos/\%7B7F04C988-4CE2-1FEA-4C54-3D05F55937FA\%7D.pdf

Bernheim, B., M. Garrett and D. Maki, (2001). Education and saving: The long term effects of high school financial curriculum mandates. Journal of Public Economics, 80(3): 435-465.

Caskey, J., C. R. Duran, and T. M. Solo (2006). "The Urban Unbanked in Mexico and the United States." Policy Research Working Paper 3835. World Bank, Washington, DC.

Child and Youth Finance International, (2012). Introducción a la educación Child and Youth: Desarrollo de la educación financiera social, y en medios de vida. Disponible en: http://childfinanceinternational.org/index.php?option=com_mtree\&task=att_download\&link_id=143\&cf_id= $\underline{200}$

CNBV. (2012). Libro blanco inclusión financiera. Comisión nacional bancaria y de valores.

CONAIF. (2013). Reporte de inclusión financiera 2013. Disponible en: https://www.google.com.mx/ webhp? sourceid =chrome-instant \&ion $=1 \&$ espv $=2 \&$ es $\quad t h=1 \&$ ie $=$ UTF8\#q=reporte $\% 20 \mathrm{de} \% 20$ inclusi $\%$ C3\%B3n\%20financiera $\% 205 \% 202013$ 


\section{NIVEL DE CONOCIMIENTO QUE TIENEN LOS ALUMNOS DE NIVEL MEDIO SUPERIOR EN TÓPICOS FINANCIEROS SOBRE GASTO Y CRÉDITO, AHORRO E INVERSIÓN Y LA ADMINISTRACIÓN DEL DINERO}

Contreras-Rodríguez, B (2016) La realidad de la educación financiera como element de inclusion.

Demirgu.-Kunt, A. y Maksimovic, V. (1998). Law, Finance, and Firm Growth. Journal

of Finance 53 (6): 2107-37.

Dupas, P., and J. Robinson (2009). Savings Constraints and Microenterprise Development: Evidence from a Field Experiment in Kenya. NBER Working Paper 14693. National Bureau of Economic Research, Cambridge, MA.

G-20 (2010). Principles for Innovative Financial Inclusion. Global Partnership for Financial Inclusion Toronto. Recuperado de: http://www.afi-global.org/sites/default/files/afi\%20g20\%20principles.pdf

G-20. (2012). Documento de discusión Presidencia Mexicana del G-20. Recuperado de: http://www.g20.utoronto.ca/2012/2012-loscabos-disc-es.pdf

Gnan, E., M. Silgoner and B. Weber, (2007). Economic and financial education: Concepts, goals and measurement. Viena: Monetary Policy \& the Economy, Q3/07, 28-44. Disponible en http://www.oenb.at/dms/oenb/Publikationen/Volkswirtschaft/Monetary-Policy-and-theE conomy/2007/Monetary-Policy-and-the-Economy-Q 3 07/chapters/mop 20073 gnan tcm16-69086.pdf

Goldsmith, R. (1959). Financial Structure and Development as a Subject for International Comparative Study. En: The Comparative Study of Economic Growth and Structure. NBER. Recuperado de: http://www.nber.org/chapters/c4417

Hair, J. F., Anderson, R. E., Tatham, R. L. y Black, W. C. (1999). Multivariate data analysis. Spain: Prentice Hall.

Hastings, J. y Tejeda Ashton, L. (2008). Financial Literacy, Information, and Demand Elasticity: Survey and Experimental Evidence from Mexico. Documento de trabajo de la Oficina Nacional de Investigaciones Economicas n.o 14538, Oficina Nacional de Investigaciones Economicas, Cambridge, MA. Recuperado de: http://www-wds.worldbank.org/external/ default/WDSContentServer/WDSP/IB/2013/07/31/000456286 20130731120207/Rendered/PDF IACS46980WP0SPA00Box379792B00PUBLIC0.pdf

King, R.G, y Levine, R. (1993). Finance and Growth: Schumpeter might be right. Journal of Economics, Vol. 108, No. 3, pp. 717-737.

Levine, R., Loayza, N., Beck, T. (2000). Financial intermediation and growth: causality and causes. Journal of Monetary Economics 46(2000), 31-77.

Leyshon, A., \& Thrift, N. (1995). Geographies of financial exclusion: financial abandonment in Britain and the United States. Transactions of the Institute of British Geographers, 312-341.

Lusardi, A. y Mitchell, 0. (2006). Financial literacy and retirement preparedness: Evidences and implications for financial education. Business Economics, National Association for Business Economics. 35-44. Recuperado de: http://www.dartmouth.edu/ alusardi/ Papers/Financial Literacy.pdf

Lusardi, A. (2008). Household Saving Behavior: The Role of Financial Literacy, Information, and Financial Education Programs. Business Economics, National Association for Business Economics. Recuperado de: http://www.nber.org/papers/w13824

Lusardi, A., O. Mitchell and V. Curto, (2009). Financial literacy among the young: Evidence and implications for consumer policy. The national bureau of economic research.

Lusardi, A. (2010). Financial Literacy among the Young: Evidence and Implications for Consumer Policy. $\quad$ Recuperado de: http://www.dartmouth.edu/ alusardi/Papers /Financial_literacy young.pdf

Lusardi, A. y Mitchell, 0. (2011). Financial Literacy and Planning: Implications for Retirement 
Wellbeing. Business Economics, National Association for Business Economics. Working Paper 17078, May 2011. Recuperado de: http://www.nber.org/papers/w17078

Mandell, L. (2008). The financial literacy of young american adults. Jumpstart coalitions survey. Recuperado de: http://www.jumpstart.org/assets/files/2008SurveyBook.pdf

Mandell, L. (2009). The impact of financial literacy education on subsequent financial behavior. Association for Financial Counseling and Planning Education. Disponible en http://6aa7f5c4a9901a3e1a1682793cd11f5a6b732d29.gripelements.com/pdf/lewis_mandell_li nda_schmid_klein.pdf

Mehrotra, N., Puhazhendhi, V., Nair, G. y Sahoo, B. (2009). Financial inclusion - an overview. National Bank for Agriculture and Rural Development No. 8121, Mumbai - 400051

Moreno-Garcia, E., García-Santillán, A., y Munguia-Tiburcio, J.P. (2013). Some aspects about financial knowledge of undergraduate students. International Journal of Management and Sustainability. 2(4):40-62.

Presidencia de México. (2007). Plan Nacional de Desarrollo 2007-2013. Pp 97-102.

Presidencia de México. (2013). Plan Nacional de Desarrollo 2013-2018. Pp 41-53, 115-122.

Ruiz, R., H., (2011). Conceptos sobre educación financiera. México: Observatorio de la economía latinoamericana, no144, disponible en http://www.eumed.net/cursecon/ecolat/mx/2011/hrr.htm

Sarma, M. (2010). Index of financial inclusión. Centre for International trade and development. India. Recuperado de: http://www.jnu.ac.in/sis/citd/DiscussionPapers/DP05_2010.pdf

Sinclair S. P. (2001). Financial exclusion: An introductory survey. Edimburgo: Universidad HeriotWatt. Report of Centre for Research in Socially Inclusive Services.

Tzu-Chin, M., Suzanne, B., Fox, J., y Cravener, G. (2007). The impact of personal finance education delivered in high school and college courses. Journal of Family and Economic, 28: 265-284. Doi: 10.1007/s10834-007-9058-7

1 Datos consultados en Banco Mundial (13 de noviembre de 2014) https://openknowledge.worldbank.org/bitstream/handle/10986/16238/9780821399859.pdf?sequence $=4$

2 Información más amplia en "Ubicando la Inclusión Financiera en el mapa mundial. Informe sobre avances de la Declaración Maya 2013".

http://www.afi-global.org/sites/default/files/publications/ afi_2013 maya_progress_report_sp_final.pdf [Consultado en octubre de 2014]

3 Se han retomado algunas de las ideas principales del discurso que dio el Dr. Agustín Carsten durante el seminario de Inclusión Financiera responsable y exitosa: Modelos Internacionales llevado a cabo en mayo del 2013 en la Cd. De México. El documento puede ser consultado desde el website: http://mx.investing.com/central-banks/bank-of-mexico/discursos/palabras-delgobernador-del-banco-de-m\%C3\%A9xico,-agust\%C3\%ADn-carstens-457 [Consultado en enero del 2014]

4 El instrumento se encuentra en el anexo 1 
